\title{
Relations between Effects and Structure of Small Bicyclic Molecules on the Complex Model System Saccharomyces cerevisiae
}

\author{
Matteo Brilli ${ }^{1 \dagger}$, Andrea Trabocchi ${ }^{2}$, Tobias Weil ${ }^{1}$, Duccio Cavalieri ${ }^{3}$ and Irene Stefanini ${ }^{4 *}$ \\ ${ }^{1}$ Centre for Research and Innovation, Fondazione Edmund Mach, Trento, Italy, ${ }^{2}$ Department of Chemistry "Ugo Schiff", \\ University of Florence, Florence, Italy, ${ }^{3}$ Department of Biology, University of Florence, Florence, Italy, ${ }^{4}$ Division of Biomedical \\ Cell Biology, Warwick Medical School, University of Warwick, Coventry, UK
}

\section{OPEN ACCESS}

Edited by:

Luis Rivas,

Consejo Superior de Investigaciones

Cientificas, Spain

Reviewed by:

Rosario Gonzalez-Muniz,

Instituto de Química Médica (CSIC),

Spain

Stefania Butini,

University of Siena, Italy

*Correspondence:

Irene Stefanin

i.stefanini@warwick.ac.uk;

stefanini.irene@gmail.com

${ }^{\dagger}$ Present Address:

Matteo Brilli,

Department of Agronomy, Food,

Natural Resources, Animals and

Environment, University of Padova,

Padova, Italy

Specialty section:

This article was submitted to Experimental Pharmacology and Drug

Discovery,

a section of the journal

Frontiers in Pharmacology

Received: 29 December 2016 Accepted: 14 March 2017

Published: 30 March 2017

Citation:

Brilli M, Trabocchi A, Weil T, Cavalieri D and Stefanini I (2017)

Relations between Effects and Structure of Small Bicyclic Molecules

on the Complex Model System

Saccharomyces cerevisiae.

Front. Pharmacol. 8:170.

doi: 10.3389/fphar.2017.00170
The development of compounds able to modify biological functions largely took advantage of parallel synthesis to generate a broad chemical variance of compounds to be tested for the desired effect(s). The budding yeast Saccharomyces cerevisiae is a model for pharmacological studies since a long time as it represents a relatively simple system to explore the relations among chemical variance and bioactivity. To identify relations between the chemical features of the molecules and their activity, we delved into the effects of a library of small compounds on the viability of a set of $S$. cerevisiae strains. Thanks to the high degree of chemical diversity of the tested compounds and to the measured effect on the yeast growth rate, we were able to scale-down the chemical library and to gain information on the most effective structures at the substituent level. Our results represent a valuable source for the selection, rational design, and optimization of bioactive compounds.

Keywords: small compounds, Saccharomyces cerevisiae, phenotypic screening, principal component analysis, stepwise regression analysis, drug screening, drug development, high-throughput screening (HTS)

\section{INTRODUCTION}

Drug discovery strongly relies on the availability of small molecule compounds bearing biological activity (Smietana et al., 2016). During the last decades, several approaches have been adopted aiming at the exploration and exploitation of the chemical variability (Richard et al., 2008). Among these, a noteworthy contribution was given by Diversity-Oriented Synthesis (DOS), which allowed the generation of the widest diversity and complexity from a simple initial scaffold (O'Connell et al., 2013). Large libraries generated with a DOS approach are a valuable resource to explore the effect of small structural changes on the biological activity of a compound (Bajorath, 2013). Once a large set of compounds is synthesized, an efficient and high-throughput assay is necessary to assess the library bioactivity (Ghannoum and Rice, 1999; Huang et al., 2004). Both target-oriented and chemical genetics-oriented approaches have been adopted to rapidly and efficiently test the biological activity of large sets of compounds (Cong et al., 2012). While target-oriented screenings offer the advantage of an a priori knowledge of the entity affected by the tested compound, chemical genetics approaches show other advantages. As

Abbreviations: BTAa, Bicycles from Tartaric acid and Amino acids; ODst, Optical Density at the culture stationary phase; YPD, Yeast Peptone Dextrose; DMSO, DiMethyl SulfOxide; PCA, Principal Component Analysis; RDA, Redundancy Analysis; AIC, Akaike Information Criterion. 
an example, cell- or organism- based screenings allow evaluating the biological function of the tested compounds without the preselection of a specific target, hence allowing the discovery of new therapeutic targets (Schenone et al., 2013). For the same reason, chemical genetics is useful for discovering compounds active against "undruggable diseases," associated with complex interactions of different biological entities (i.e., protein-protein, protein-DNA), for which targeted-oriented approaches can not be adopted (Altmann et al., 2009). Furthermore, the cellular context of chemical genetics allows to assessing whether the efficacy of the compound under study is reduced by metabolic reactions or by physical cellular barriers (i.e., the cell membrane) (Swinney and Anthony, 2011). All these advantages made chemical genetics the most successful approach for evaluating the activity of small-molecule libraries (Swinney, 2013). The downside of such phenotypic screenings is that they allow the identification of active compounds, but further studies are necessary afterward to identify their targets (Schenone et al., 2013).

The budding yeast Saccharomyces cerevisiae is mainly used for wine, beer, and bread fermentations but is also suitable for high-throughput screening approaches (Schenone et al., 2013). In fact, it has been successfully used as a model organism in phenotypic screens aimed at the identification of molecules of pharmacological interest (Brenner, 2004; Hoon et al., 2008). Among the others, a matter of clinical relevance could largely benefit from the testing of parallel synthesis product on the budding yeast: systemic fungal infections, which have been estimated to cause more casualties than malaria (http://www.bbc. co.uk/news/health-36702215). Because of the similarity between $S$. cerevisiae and pathogenic fungi, compounds inhibiting the growth of the former are also potentially active against $A$. fumigatus, C. albicans and other important human pathogens (Goldstein and McCusker, 2001). By testing the chemical variability offered by DOS libraries on Saccharomyces cerevisiae it is therefore possible to assess the relationship between chemical variance and biological activity and get insights about much more complicated systems.

Here we dissected the relations between the $S$. cerevisiae phenotypic growth effects and chemical structures of 101 small bicyclic compounds. The molecules object of this study were generated by combining different functional groups with three scaffolds: BTAa (Bicycles from Tartaric and Amino acids), BTK (Bicycles from Tartaric and amino ketones) and BTG (Bicycles from Tartaric acid and Glyceraldehyde derivatives). In a previous study from our group (Stefanini et al., 2010), we evaluated the effect of these compounds on the growth rate of several S. cerevisiae strains. This study allowed the selection of promising antifungal compounds, one of which (named 089) was the most effective in reducing the yeast growth. In the present study, we took advantage of the high degree of chemical diversity of the tested compounds and the measured effects on the yeast growth rate to scale-down our chemical library and to gain information on the most effective (combinations of) substituents providing a valuable source for the rational design of bioactive compounds and their optimization.

\section{MATERIALS AND METHODS}

\section{Compounds}

The tested library consisted of BTAa (Bicycles from Tartaric acid and Amino acids), BTK (Bicycles from Tartaric acid and amminic ketones) and BTG (Bicycles from tartaric acid and glyceraldehyde derivatives) compounds (Trabocchi et al., 2008). BTAa were synthesized from 3-aza-6,8-dioxa-bicycle[3.2.1]octane as the fundamental structure and variously functionalized as previously described (Machetti et al., 2007). Stability of the ketal moiety over strong aqueous acid conditions has been assessed by several applications of these compounds in peptide synthesis. For an account of all the chemical features of these compounds, please refer to Trabocchi et al. (2006). For uniformity, all molecules were dissolved in DMSO at the same stock concentration $(5 \mathrm{mM})$ and were compared to the same control (DMSO in volumes equal to the volume of the treatment molecule). Molecule structures are shown in Supplementary Figure 1.

\section{Yeast Strains, Culturing and Measurement of Phenotypic Effects}

The Saccharomyces cerevisiae strains used in this study are: the wild type strains BY4742 and W303, and three BY4742 deletion strains, $\Delta$ erg6, $\Delta$ pdr3, and $\Delta$ snq2. Yeast cells were grown in liquid YPD medium (2\%Yeast extract, $1 \%$ peptone, $2 \%$ Dextrose) at $27^{\circ} \mathrm{C}$ with orbital shaking. Before treatment, yeast cells were pre-cultured overnight. Cells were then inoculated at $5^{*} 10^{\wedge} 5$ cells $/ \mathrm{ml}$ in liquid YPD supplemented with either the molecule $(0.3 \mathrm{mM})$ or DMSO (equal volumes, see details in the previous section). The effects induced by molecules on $S$. cerevisiae growth were summarized as the difference of the Optical Density at the stationary phase (ODst) of the treated culture compared to the control (treated with DMSO) and expressed as a percentage of the ODst of the untreated culture. The ODst percentages calculated for each molecule on the tested strains are listed in Supplementary Table 1.

\section{Principal Component Analysis}

Principal component analysis (PCA) was carried out by using as cases the 19 molecules selected as reducing the ODst of the wild-type S. cerevisiae W303 strain and as descriptors (variables) both the chemical characteristics (substituents at the R1, R2, R3, R4, and R4" positions and molecule scaffolds) and the phenotypic effects (ODst \%) on tested strains. Since variables encompassed both qualitative (chemical groups) and quantitative (phenotypic effects) descriptors, we used the dudi.mix function of the $\mathrm{R}$ package ade4 (Dray and Dufour, 2007), combining PCA for the quantitative factors and MCA (Multiple Correspondence Analysis) for qualitative ones (see ade4 $\mathrm{R}$ package documentations for further details, Dray and Dufour, 2007).

\section{Redundancy Analysis}

To study the relevance of the molecules' general features (other than chemical substituents) on the biological effect, we explored the relationship of chemical hindrance and polarization with the phenotypic effects of the molecule. We performed redundancy 
analysis (RDA, rda function of the vegan $\mathrm{R}$ package, Oksanen et al., 2016) to examine how much variation in molecular features explain the variation in the phenotypic effect. In particular, RDA was carried out on two separate matrices, one including the phenotypic effects of the 19 selected molecules on the complete set of strains, the other including the chemical features (either the polarizability or the hindrance calculated for the whole molecule or the substituents separately). Molecules and chemical moieties polarizabilities were calculated using the Marvin Sketch software (5.10.0) and the tendency of the analyzed entity to diminishing the external electric field was calculated with a method based on the Thole's parameters (Jensen et al., 2002). Similarly, molecules and substituents steric hindrances were calculated by using the Marvin Sketch software (5.10.0) and evaluating the van der Waals volume of the conformer (in Armstrong3) (Jensen et al., 2002). Molecular and substituents hindrance and polarizability values are listed in Supplementary Table 1.

\section{Stepwise Regression Analysis}

Stepwise regression analysis was carried out to identify the chemical variables (substituents or scaffolds) which are most responsible for the biological effect observed in experiments. Molecules functionalizations and scaffolds were translated into dummy variables indicating their presence or absence. Stepwise regression analysis was carried out on molecules inducing an ODst $>2 \%$ or $<-2 \%$ on the wild-type W303 strain compared to the ODst of the control culture using the stepAIC function of the MASS package (Venables and Ripley, 2002). Stepwise regression analysis consisted in the assessment of linearity among the biological effect (ODst on W303 strain) and the chemical structure tested by adding or removing one-by-one the variables and evaluating whether the change improved the model. Briefly, the dummy variables indicating the presence/absence of substituents and scaffolds were grouped according to their position (in case of substituents) or type (for scaffolds). Because stepwise regression analysis works by adding or removing one variable per step, the most complex model encompassed one dummy variable for each group, and the most simple model encompassed a dummy variable for one group only. Each modification of the variable set used to generate the model represented a single step. For each step, the resulting model was evaluated using the AIC (Akaike Information Criterion). The best model was considered the one with the highest AIC. The substituents that appeared only once in the chemical library were removed from the dataset to avoid the generation of misleading models.

\section{RESULTS}

\section{Evaluation of the Biological Activity of the Chemical Library}

The chemical diversity of the 101 analyzed compounds (molecular formula in Molecular_formula_strings.csv, drawings in Supplementary Figure 1) stems from three BTAa (Bicycles from Tartaric acid and Amino acids) scaffolds, combined with different R1, R2, R3, and R4' - $^{\prime \prime}$ appendages (Figure 1) (Stefanini et al., 2010). All BTAas are derived from tartaric acid and one

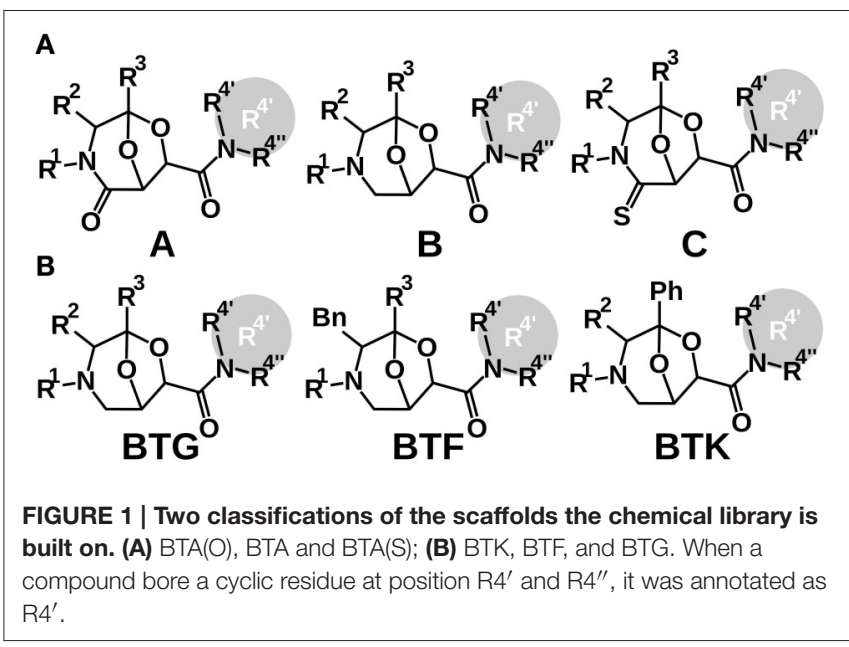

amino acid (Guarna et al., 1999; Trabocchi et al., 2002, 2003; Cini et al., 2006; Machetti et al., 2007). BTAas can be classified in two ways: according to the absence/presence of a double bonded Oxygen or Sulfur (Scaffold B, A, and C, Figure 1A) (Guarna et al., 1999), or according to the chemical reactants used to synthesize them (Figure 1B) (Machetti et al., 2007).

In our previous study (Stefanini et al., 2010), we observed that only 30 of the 101 compounds composing the tested library reduced the Optical Density at the stationary phase (ODst) of the wild-type Saccharomyces cerevisiae W303 strain when tested at a $0.3 \mathrm{mM}$ concentration. To explore the drug potential of the tested compounds against the widest range of fungal and mammalian cells, it is pivotal to elude defenses specific of the organism used as a model to test the molecule bioactivity. Aiming at this, the selected compounds were further tested on a set of strains deleted in genes known to participate in an accurate and advanced response to drugs that characterizes $S$. cerevisiae and a few other fungi (Wolfger et al., 2001). In particular, the activity of the selected compounds was evaluated in strains deleted in either ERG6, SNQ2, or PDR3 genes and in the corresponding wild-type BY4742 strain as a reference. In our previous study, this round of phenotypic assays allowed us to identify, among the 30 molecules most active in the W303 strain, 19 compounds decreasing the ODst of the test strains by more than $20 \%$, hence representing good candidates as fungicides (Stefanini et al., 2010).

\section{Identifying the Relations between Molecules' Chemical Variability and Susceptibility to Yeast Defenses}

Exploring the relations between presence/absence of specific substituents or scaffolds and the effects induced on the set of deletion strains is helpful to understand which chemical features make the compound unaffectable by the yeast response to drugs. Aiming at this, we applied Principal Component Analysis (PCA) on the effects of the 19 molecules tested on the complete set of $S$. cerevisiae strains to explore the relations among chemical entities and the effects on the different strains (Figure 2). This analysis, carried out combining PCA for quantitative variables (the ODst values) and MCA 


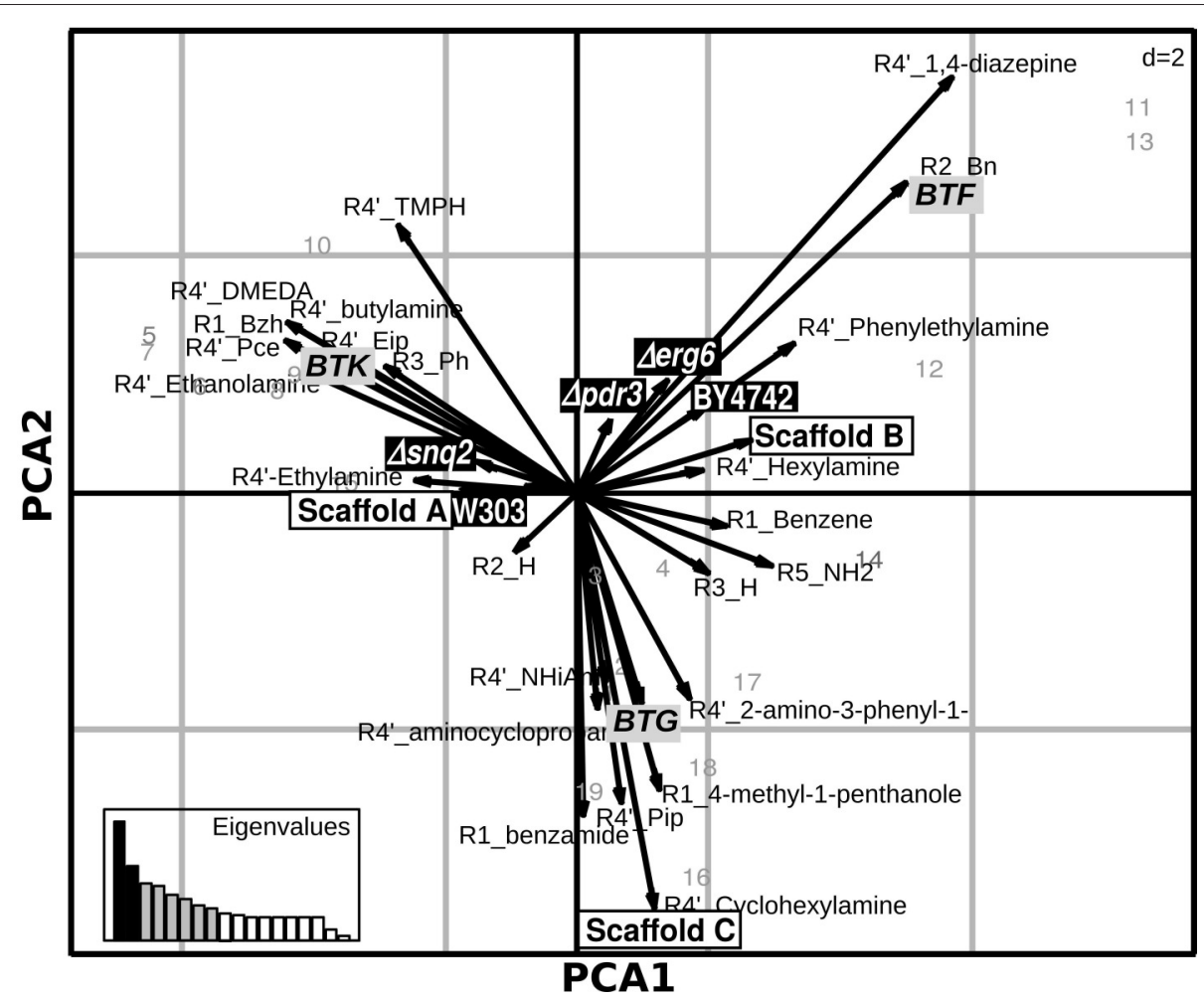

FIGURE 2 | Relationship among chemical features of selected compounds and their activity. Duality diagram for the first two principal components obtained by analyzing correspondence between the $\triangle \mathrm{ODst} \%$ and the chemical features of the molecules selected at the first-level. The cases for analysis were the molecules selected in the first level assay as able to induce a W303 ODst decrease higher than the $20 \%$ with respect to the ODst of the untreated culture. The variables are both the $\triangle$ ODst \% induced by the molecules on the tested strains and the molecule chemical features (substituent types, scaffold types); black-boxes labels: effects on the tested strains, white-boxes labels: molecules classification as shown in Figure 1A, gray-boxes labels: molecules classification as shown in Figure 1B. PCA1, First Principal Component; PCA2, Second Principal Component.

(Multiple Correspondence Analysis) for qualitative variables (the scaffold and the R substituent at different positions), allows to exploring associations among variables measured for the library compounds (cases). The molecules inducing the highest ODst reduction on the W303 wild-type strain also proved to induce opposite effects on the deletion $\Delta$ erg 6 and $\Delta$ pdr3 strains (as indicated by the corresponding arrows pointing to opposite directions in Figure 2). The effects induced on strains BY4742, $\Delta$ erg6 and $\Delta$ pdr3 were strongly correlated with the BTF class of compounds, while the other two classes of compounds were not correlated with any of the tested strains (Figure 2). In general, the BY4742 strain was less affected by the treatments than W303 (Figure 3A). The presence of either 1,4-diazepine or phenylethylamine at position $\mathrm{R} 4$ " and of benzene at R2, mainly associated with the BTF class, was correlated with the effect induced on the deletion strains $\Delta$ erg6 and $\Delta$ pdr3 and on the wild-type BY4742 strains (Figure 2). The presence of ethylamine at position $\mathrm{R} 4$ " was correlated to the effects induced on the wild-type strain W303 and on the deletion strain $\Delta$ snq2 (Figure 2). The effects induced on the $\Delta$ snq2 strain by the compounds based on scaffold A were more variable compared to those induced on other strains, comprising both different levels of inhibition and increase of yeast growth (Levene test,
$P=0.005)$. Yet they showed an overall trend toward being more beneficial than all other compounds (Levene test, $P=0.005$ ) as they induced a larger increase of culture ODst when compared to the control or to molecules of the B scaffold (Figure 3B). On the contrary, compounds built around scaffold $\mathrm{B}$ induced variable and beneficial effects on the wild-type BY4742 strain (Levene test, $P=0.025$, Figure 3B). Different scenarios were observed when considering the effects induced by the selected molecules classified in terms of their scaffolds classified as BTG, BTF, or BTK (Figures 3C-E). Here, only BTF compounds induced proportional effects on both the $\Delta$ erg6 and the BY4742 wild-type strains, while BTKs just had a slight effect on the growth of the BY4742 strain, and BTG molecules induced opposite effects on the wild-type and on the deletion strains -weak ODst decrease in the first and ODst increase in the latter- (Figure 3A). No other significant differences were observed when comparing the effects of the molecules belonging to the $\mathrm{A}, \mathrm{B}$, or $\mathrm{C}$ classes (Supplementary Figure 2) nor among the BTG, BTK, or BTF molecules (Supplementary Figure 3).

Since steric hindrance and polarizability of the molecule (or parts of it) play a pivotal role in the compound's druggability (the ability of the drug to bind its target and exploit its activity), we explored the existence of a relationship among these two 


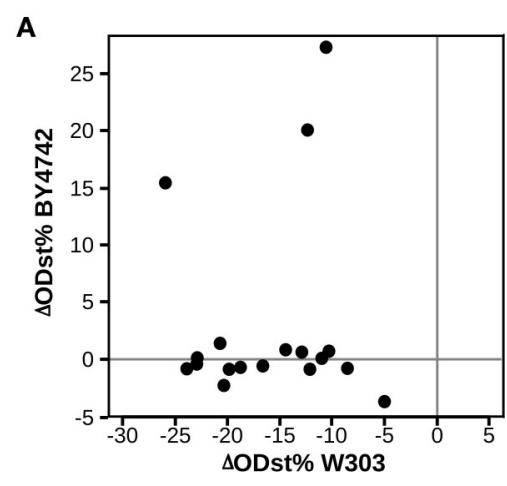

C
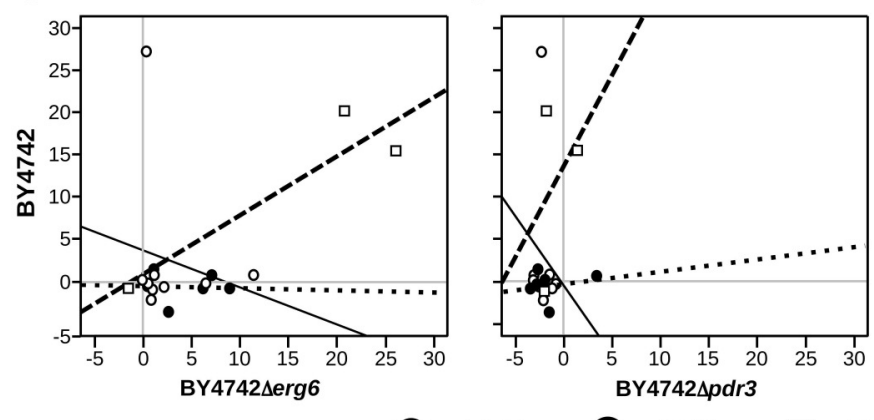

B

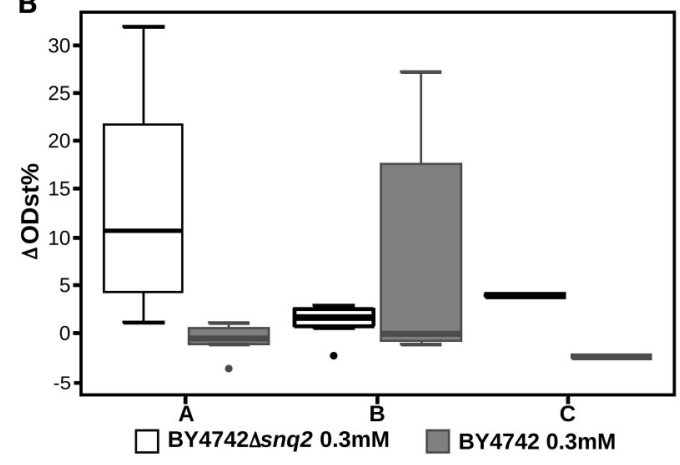

E

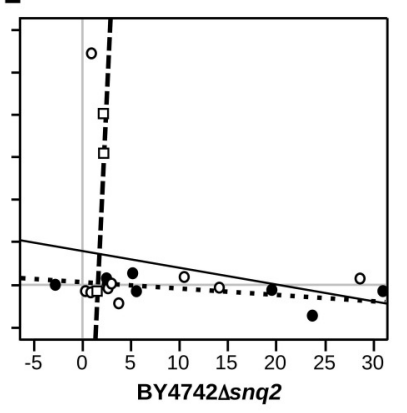

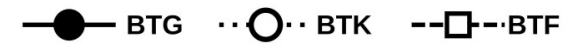

FIGURE 3 | Highlights of the effects of the selected molecules and their chemical characteristics. (A) Direct comparison of the molecules effect on BY4742 and W303 strains, each dot indicate a different molecule; (B) boxplot showing the effects on the BY4742 $\Delta$ snq2 strain culture ODst caused by the molecules grouped according to the scaffold classification showed in Figure 1A; (C-E) relationship between the effects induced by the molecules classified accordingly to Figure 1B on the wild-type BY4742 and on the $\Delta$ erg6, $\Delta \mathrm{pdr} 3$, and $\Delta$ snq2 strains, respectively.

variables and the biological effects induced on the different strains. Aiming at this, we applied Redundancy Analysis to explore how much variation in molecular features (hindrance and polarizability) explains the variation in phenotypic effects. Briefly, RDA estimates the degree to which a block of variables can predict a second set of variables by linear relationships (Varmuza et al., 2012). In our study the two "blocks of variables" were the phenotypic measures (ODst for each selected molecule in all the tested strains) and the chemical features (either the polarizability or the hindrance calculated for the whole molecule or the substituents separately). Both the hindrance and the polarizability of the group at position $\mathrm{R} 2$ resulted to be important for molecules activity (Figure 4). The higher the R2 hindrance, the more pronounced was the positive effect on $\Delta$ erg6 cell growth (Figure 4A, Supplementary Figure 4A). The molecule with the highest R2 polarizability (047) is also the molecule inducing the most beneficial effect on wild-type cells (Figure 4B, Supplementary Figure 4B). The level of polarizability of the complete molecule was related to the activity on strain $\Delta$ erg6 (Figure 4B). Indeed, the molecule less prone to polarization (066) induced the highest cell death on the $\Delta \operatorname{erg} 6$ strain (Figure 4B). On the contrary, mild molecule polarizability (053 and 038) was associated to the highest beneficial effect on the $\Delta$ erg6 strain (Supplementary Figure 4C). The same profile was shown by the relation between the hindrance of the group at position $\mathrm{R} 4^{\prime \prime}$ and the molecules effect on strain $\Delta \operatorname{erg} 6$ (Figure 4A). The molecule with the smallest $\mathrm{R} 4$ " group hindrance (066) was the one inducing the most deleterious effect on both $\Delta$ erg6 and $\Delta$ snq2 strains, while the molecules with intermediate $\mathrm{R} 4{ }^{\prime \prime}$ hindrance (053 and 038) were the most beneficial on the $\Delta$ erg6 strain (Supplementary Figure 4D).

\section{Analysis of the Complete Chemical Variance}

The lesson learned from the effects on deletion strains of a selected pool of molecules gave several indications to dissect the contribution of the chemical functions to the biological effects the molecules can induce when the $S$. cerevisiae-specific response mechanisms are impaired. We went further, aiming at the exploration of the relationship between the chemical variance disclosed by the complete library and the biological effects induced on the wild-type W303 strain. The PCA on the $\triangle$ ODst induced by the complete set of molecules revealed only weak correlations with some chemical features of the molecules (Figure 5A). A positive effect on the cell culture growth was associated to BTG molecules, to the $\mathrm{C}$ scaffold and to several chemical groups at positions R1 and R4' (the corresponding arrows pointed in the same direction of the "W303" arrow, indicating the ODst variable, Figure 5A). On the contrary, neither the chemical groups at position R2 nor R3 were associated to any particular effect on the strain growth. The W303 ODst decrease (the opposite direction of the thick 

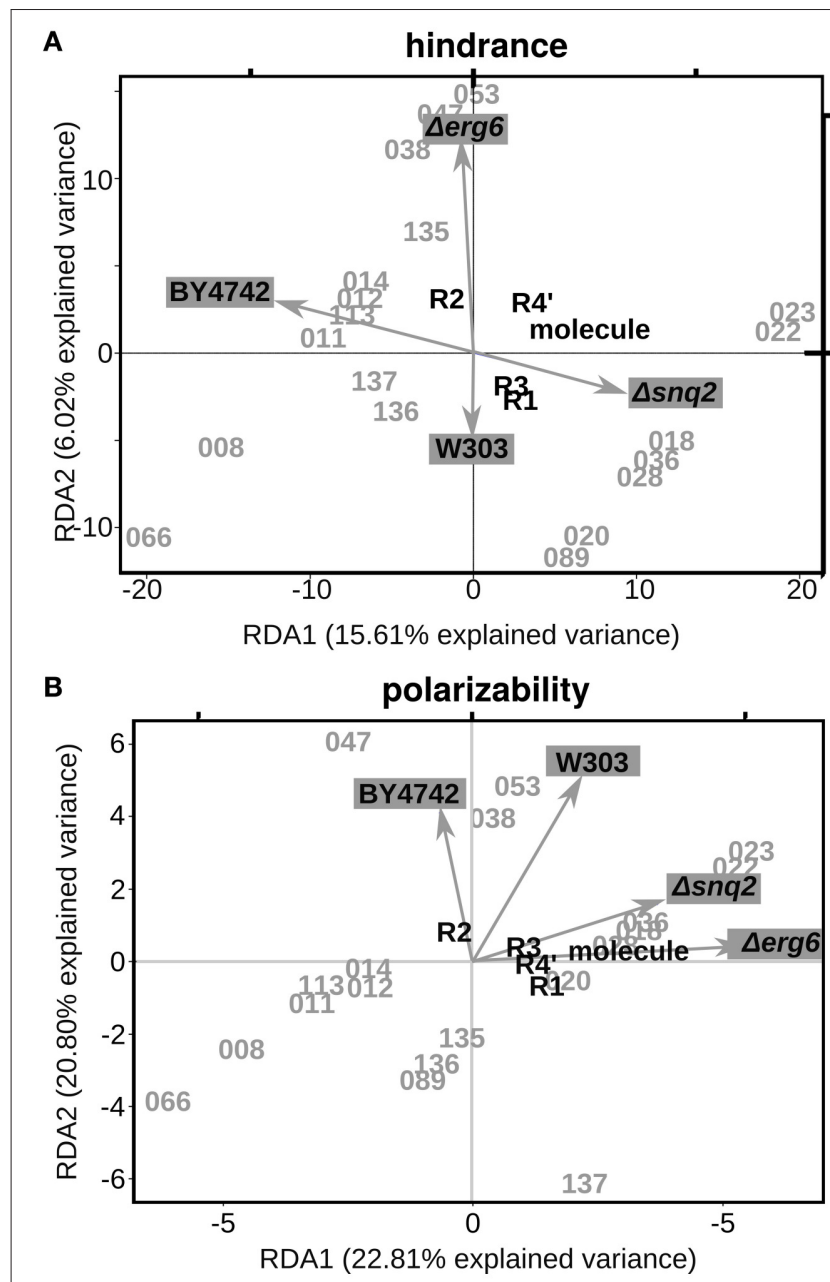

FIGURE 4 | Relationship between steric hindrance or polarizability and the phenotypic effects. (A) First two explanatory components identified with the Redundancy Analysis (RDA) on $\triangle O D s t \%$ values and steric hindrances. RDA1, First RDA constrained axis; RDA2, Second RDA constrained axis. The $\triangle$ ODst \% calculated for every strain treatment were used as constraining variables; as explanatory variables we used the steric hindrance values (A3) calculated with the Marvin Sketch software (5.10.0). (B) First two explanatory components identified with RDA on $\triangle$ ODst \% values and molecule/substituent polarizability. The $\triangle \mathrm{ODst} \%$ calculated for every strain treatment were used as constraining variables; as explanatory variables we used the polarizability indexes (A3) calculated with the Marvin Sketch software (5.10.0).

black arrow in Figure 5A) was not associated to any scaffold or BTA type, but it was weakly associated to the presence of n-propyl, n-Butyl, TMPH (thiomorpholine), DMEDA ((2aminoethyl)dimethylamine) or ethanolamine at position $\mathrm{R} 4^{\prime}$ or $\mathrm{R} 4^{\prime \prime}$. As regarding molecule functionalization, none of the chemical groups per se showed to be significantly correlated to the ODst variation (Wilcoxon test Supplementary Tables 2-4). A closer look into the PCA ordinations based on the results obtained from the complete set of molecules (Figure 5A) highlighted a trend related to the type of amide present at R4 ${ }^{\prime \prime}$ position. Indeed, a secondary amide at R4" decreased the ODst with respect to the presence of a tertiary amide. In particular, the largest difference was evident for C scaffold molecules (BTA(S),
Figure 1A), which reduced the ODst when bearing a secondary amide, whereas only slightly varied the ODst when a tertiary amide was present (Figure 5B, Wilcoxon test, $P=0.067$ ).

The biological activity of a compound may be due to either single chemical groups or a combination of them. The identification of relationships between the biological activity and the combinations of chemical groups is instrumental to infer and design compounds inducing the desired fungicide/fungistatic effect. We thus carried out a series of stepwise regressions $(19,440$ complete models in total) by exploring every possible combination of scaffold types and chemical groups at the 4 positions (avoiding the combination of more than one substituent at the same position, see materials and methods). In other words, linearity among the biological effect and the chemical structure was tested by adding or removing one-by-one the variables and evaluating whether the change improved the model as summarized by the AIC (Akaike Information Criterion, a measure of the relative quality of statistical models). Because the linear model would be strongly affected by near-to-zero ODst, we carried out the analysis on the compounds inducing an ODst $>2 \%$ or $<-2 \%$ (71 compounds, Supplementary Table 1 ). Several combinations of chemical variables gave rise to small AIC (gray in Supplementary Table 5). All of them contained scaffold A (Figure 1A), and ethylamine at R4" (gray in Supplementary Table 5); the detailed observation of the stepwise regression analysis showed that both of these molecular characteristics induced a decrease in W303 ODst (negative coefficient, Supplementary Table 6). In addition, the growth inhibition was also correlated with the presence of benzene at the R2 position (Supplementary Table 6, Supplementary Figure 5). Neither the molecule type (BTG, BTK, or BTF, Figure 1B) nor the type of substituent at R1 or R3 had a significant influence on the ODst. The compound showing the relevant chemical features revealed by the means of stepwise regression analysis (compound 089) resulted to be also the strongest growth inhibitor among the 19 molecules selected in the previous study after extensive experimental characterizations. This compound is a BTK build on the A scaffold with benzhydryl at position R1, a $\mathrm{CH} 2$ moiety at position R2, a phenyl at position R3 and an ethylamine at position $\mathrm{R} 4$ ". The comparison of the effects on the wild-type W303 strain induced by molecules identical to 089 for all but one chemical group revealed interesting information (Figure 6). The substitution of the scaffold (024, scaffold A) reduced only slightly the activity of the compound (from $-22.8 \%$ to $-17.60 \%$ ). Instead, the substitution of the $\mathrm{R} 4^{\prime \prime}$ ethylamine with other chemical groups reduced the activity of the compound from -22.8 to $-13.8 \%$ (089 and 103 , respectively), or even induced the opposite effect (100 and 099 are associated to a 1.5 and $5.1 \%$ ODst, respectively) (Figure 6).

\section{DISCUSSION}

The present analysis has been carried out on an entire organism, a complex system, thus the full dissection of the contribution of the chemical functions to the effects was highly complex too. Nevertheless, the combination of biological and chemical 
A

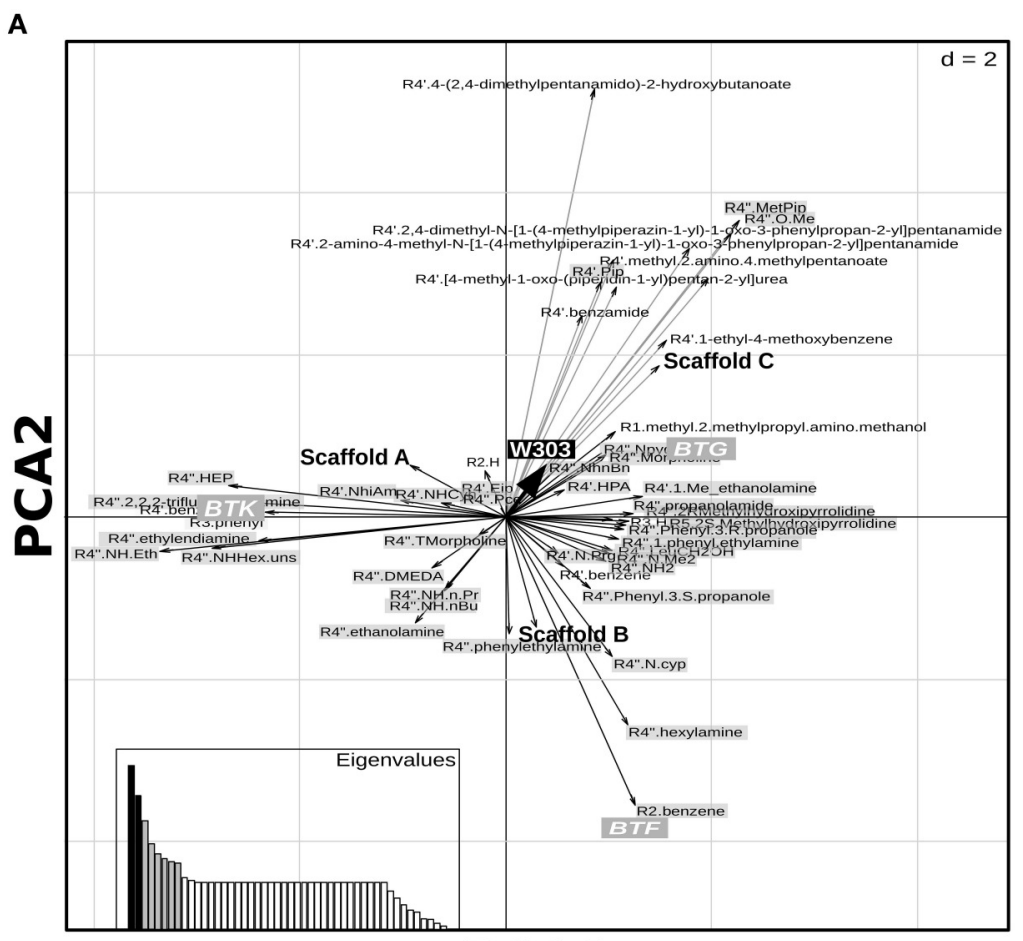

PCA1
B

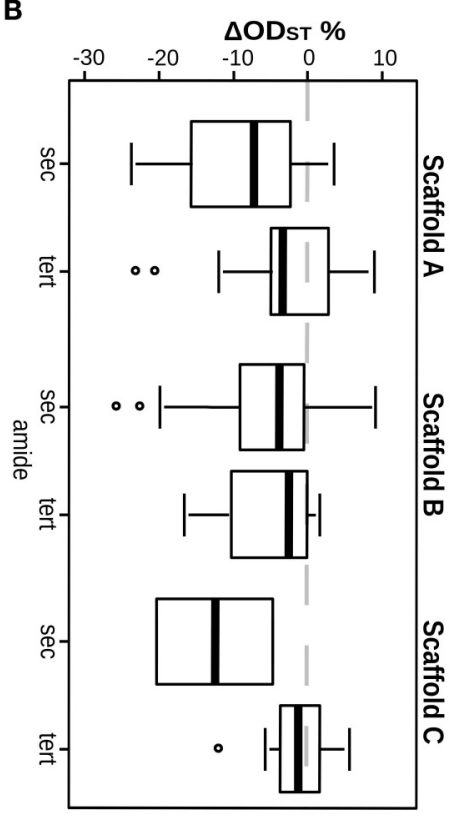

FIGURE 5 | Results of the analysis on the complete library of molecules. (A) Duality diagram for the first two principal components obtained by analyzing the $\Delta$ ODst $\%$ and the chemical features of the complete library of molecules. The cases for analysis were the molecules composing the library. The variables are the $\triangle$ ODst \% induced by the molecules on the W303 strain and the molecule chemical features (substituent types, scaffold types); black-box labels: effects on the tested strains, bold labels: molecules classification as shown in Figure 1A, gray-box labels: molecules classification as shown in Figure 1B. (B) Boxplots representing the influence of the presence of a secondary or tertiary amine in position R4" in the effects induced on the W303 wild-type strain ODst by the molecules classified in three groups as described in Figure 1A.

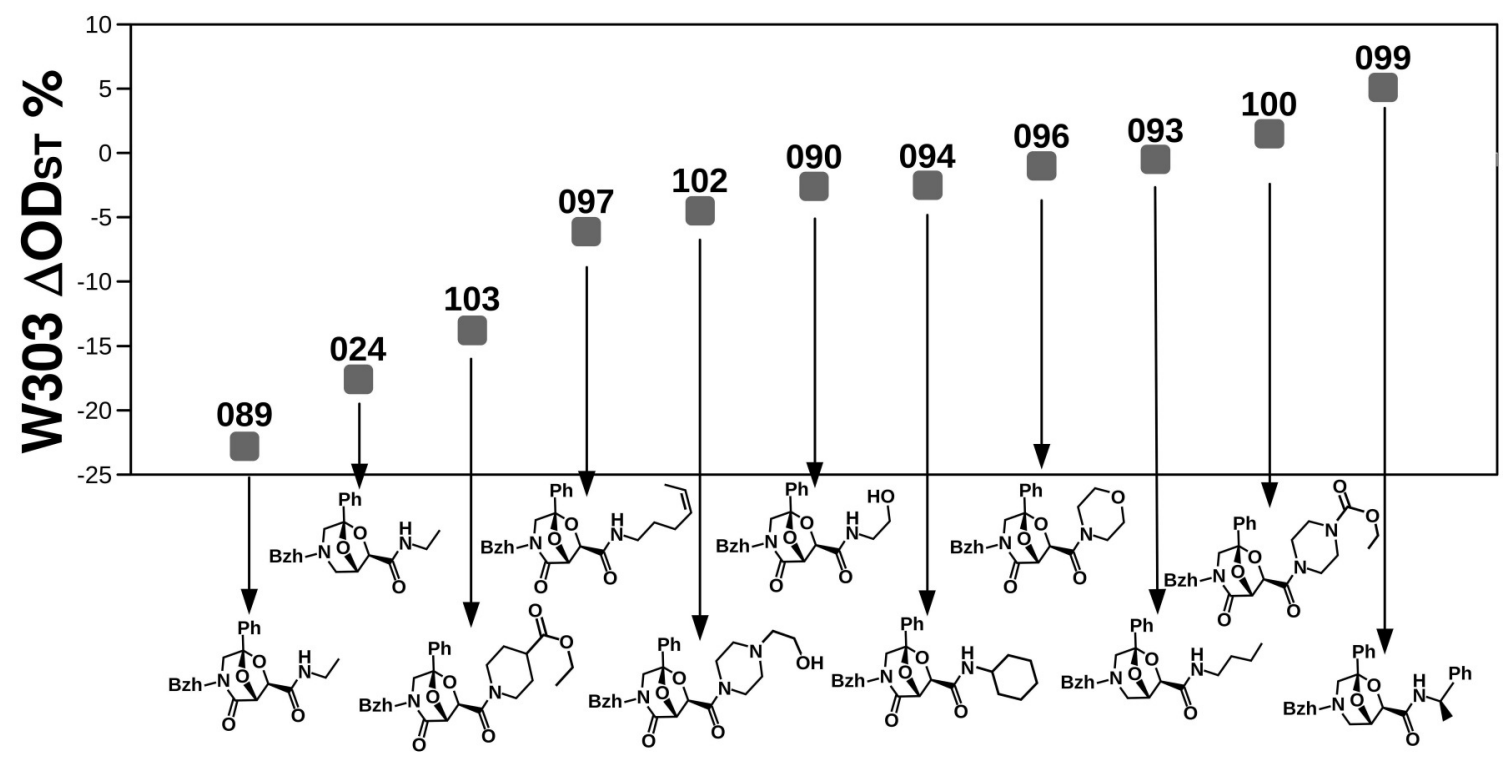

FIGURE 6 | Comparison of the effects induced on the W303 wild-type by the most fungicide molecule with respect to molecules almost identical to it but for small chemical features. Numbers correspond to compound names. 
variances allowed us to draw general indications. The present study was not aimed at identifying the chemical features optimizing a specific mechanism of action. Rather, this organismlevel analysis revealed valuable information that can be used to ameliorate the molecule chemical structure aiming at the optimization of the effect on the growth rate of $S$. cerevisiae. Irrespective of the drug chemical structure, the W303 strain was more sensitive than BY 4742 to the treatment with BTAa compounds. These two wild-type strains mainly differ for the presence in BY4742 of a malfunctioning HAP1 gene reducing the BY4741 respiration. The higher sensitivity of W303 may therefore suggest that respiratory metabolism is involved in the pathway targeted by these molecules. By exploring the relations among structures and phenotypes for only the compounds selected as inducing the greatest effect on the wild-type W303 strain, we obtained objective information, which could not be summarized by visually inspecting the phenotypic results and the chemical structures. The classification of the tested molecules into three groups based on the absence/presence of a double bonded Oxygen or Sulfur (Scaffold B, A, and C, respectively) allowed to uncover the backbones' potential. While the presence of a sulfur atom on the principal ring [BTA(S), scaffold C, Figure $1 \mathrm{~A}$ ] made the molecule more prone in enhancing growth, two different (but still unknown) mechanisms of action can be hypothesized for the BTA(O) (scaffold A) and BTA (scaffold B). Molecules based on scaffold A generate a benefit to the cell, but this effect is avoided by their extrusion by the cell due to the Snq2 protein. On the contrary, wild type cells benefit from molecules built on scaffold B (BTA), but the this effect is not observed when Snq2p is missing. This could indicate that the molecules built on scaffold B influence cell metabolism by increasing respiration. Indeed, Snq2p requires ATP to extrude the drugs, which may explain the respiration request (Dueñas-Sánchez et al., 2010). S. cerevisiae can both breathe and ferment, but it has been shown that respiration increases biomass production (Decottignies et al., 1995). Thus, by producing more ATP and consuming more oxygen, the biomass increases, similar to the induction observed during the treatment of the wild type cells with the molecules build on the B scaffold. On the contrary, the lack of the Snq2 protein reduces ATP request, thus reducing the biomass production and restoring the situation of the untreated control upon the treatment with compounds bearing the $\mathrm{B}$ scaffold. A different story can be depicted when comparing molecules classified as BTF, BTK, and BTG. The BTG class of molecules (generated by the combination of tartaric acid and glyceraldehyde derivatives) was the only one not modifying the ODst of the wild-type BY4741 but increasing the ODst of the deletion strains. This result clearly indicates that the (beneficial) activity of these molecules in the BY4741 genetic background is affected by the yeast response/resistance to drugs. Indeed, cell growth is improved by this class of molecules only when the cell lacks its defense systems. Yet, the molecules bearing the phenyl group at R3 (BTKs) were the less affected by the yeast drug response, and induced effects having the same trend on both deletion and wild-type strains. The known drug response machinery does not affect the activity of these molecules. Several characteristics of the molecules, in particular those involving the R2 and R4" positions, are important for biological activity.
Since bulkier groups at position R2 are associated with a more beneficial effect on strains having a lax cell wall $(\Delta$ erg6), they probably promote binding or adsorbing of the molecule on the cell wall. Moreover, if these groups are inferred to be more polarizable, the molecules are more beneficial to the wild-type strain than to deletion strains, probably because of a less efficient detoxification by the cell or due to a higher ability of the molecule to enter into the cell. Even more relevant is the chemical group present at position $\mathrm{R} 44^{\prime \prime}$. The groups at this position, being the most variable in the chemical library studied, clearly showed that several features have to be taken into account when trying to improve the activity of a certain molecule. The high deleterious effect induced on $\Delta$ erg6 cells by compounds having high steric hindrance at $\mathrm{R} 4^{\prime \prime}$ can be ascribable to the fact that those molecules are able to permeate the cell wall because of the defect in the ergosterol biosynthesis pathway associated with this deletion. A secondary amide in this position makes the deleterious effect even more pronounced. Indeed, the fungicide compound with the highest activity of our chemical library had an ethylamine at $\mathrm{R} 4{ }^{\prime \prime}$, and induced the highest rate of ODst reduction in all tested strains.

By analyzing the whole set of compounds, we were able to identify the chemical features (substituents or scaffolds) which are most responsible for the biological effect. We further show that results obtained with a thorough biological screen carried out on deletion strains (whose data were not used to train the models) highly overlap with those coming from a preliminary screen that was followed by stepwise regression. Despite suggesting to synthesize and test in vivo novel compounds was outside the scope of this work, we showed that the regression approach can help in guiding molecular testing on those molecules that are predicted to be more active. Thus, this simple strategy can be successfully exploited for the unbiased identification of a subset of most active constituents from the screening of very large compound libraries.

\section{AUTHOR CONTRIBUTIONS}

MB and IS conceived and coordinated the study and wrote the paper. IS performed the analyses. AT synthesized the molecules, provided technical assistance and contributed in discussing the results. TW and DC contributed in discussing the results. The manuscript was written through contributions of all authors. All authors have given approval to the final version of the manuscript.

\section{FUNDING}

IS was supported by a Fellowship from the Wellcome-Warwick Quantitative Biomedicine Programme.

\section{SUPPLEMENTARY MATERIAL}

The Supplementary Material for this article can be found online at: http://journal.frontiersin.org/article/10.3389/fphar. 2017.00170/full\#supplementary-material 


\section{REFERENCES}

Altmann, K. H., Buchner, J., Kessler, H., Diederich, F., Krautler, B., Lippard, S., et al. (2009). The state of the art of chemical biology. Chembiochem 10, 16-29. doi: $10.1002 /$ cbic. 200800758

Bajorath, J. (2013). "Structure-activity relationship data analysis: activity landscapes and activity cliffs," in Diversity-Oriented Synthesis: Basics and Applications in Organic Synthesis, Drug Discovery, and Chemical Biology, ed A. Trabocchi (Hoboken, NJ: John Wiley and Sons), 507-531.

Brenner, C. (2004). Chemical genomics in yeast. Genome Biol. 5:240. doi: 10.1186/gb-2004-5-9-240

Cini, N., Danieli, E., Menchi, G., Trabocchi, A., Bottoncetti, A., Raspanti, S., et al. (2006). 3-Aza-6,8-dioxabicyclo[3.2.1] octanes as new enantiopure heteroatomrich tropane-like ligands of human dopamine transporter. Bioorg. Med. Chem. 14, 5110-5120. doi: 10.1016/j.bmc.2006.04.019

Cong, F., Cheung, A. K., and Huang, S. M. (2012). Chemical genetics-based target identification in drug discovery. Annu. Rev. Pharmacol. Toxicol. 52, 57-78. doi: 10.1146/annurev-pharmtox-010611-134639

Decottignies, A., Lambert, L., Catty, P., Degand, H., Epping, E. A., MoyeRowley, W. S., et al. (1995). Identification and characterization of SNQ2, a new multidrug ATP binding cassette transporter of the yeast plasma membrane. J. Biol. Chem. 270, 18150-18157. doi: 10.1074/jbc.270.30. 18150

Dray, S., and Dufour, A. B. (2007). The ade4 package: implementing the duality diagram for ecologists. J. Stat. Softw. 22, 1-20. doi: 10.18637/jss.v022.i04

Dueñas-Sánchez, R., Codón, A. C., Rincón, A. M., and Benítez, T. (2010). Increased biomass production of industrial bakers' yeasts by overexpression of Hap4 gene. Int. J. Food Microbiol. 143, 150-160. doi: 10.1016/j.ijfoodmicro.2010.08.009

Ghannoum, M. A., and Rice, L. B. (1999). Antifungal agents: mode of action, mechanisms of resistance, and correlation of these mechanisms with bacterial resistance. Clin. Microbiol. Rev. 12, 501-517.

Goldstein, A. L., and McCusker, J. H. (2001). Development of Saccharomyces cerevisiae as a Model Pathogen: a system for the genetic identification of gene products required for survival in the mammalian host environment. Genetics $159,499-513$.

Guarna, A., Guidi, A., Machetti, F., Menchi, G., Occhiato, E. G., Scarpi, D., et al. (1999). Synthesis and reactivity of bicycles derived from tartaric acid and a-amino acids: a novel class of conformationally constrained dipeptide isosteres based upon enantiopure 3-Aza-6,8-dioxabicyclo[3.2.1] octane7-carboxylic Acid. J. Org. Chem. 64, 7347-7364. doi: 10.1021/jo99 04967

Hoon, S., St Onge, R. P., Giaever, G., and Nislow, C. (2008). Yeast chemical genomics and drug discovery an update. Trends Pharmacol. Sci. 29, 499-504. doi: 10.1016/j.tips.2008.07.006

Huang, J., Zhu, H., Haggarty, S. J., Spring, D. R., Hwang, H., Jin, F., et al. (2004). Finding new components of the target of rapamycin (TOR) signaling network through chemical genetics and proteome chips. Proc. Natl. Acad. Sci. U.S.A. 101, 16594-16599. doi: 10.1073/pnas.0407117101

Jensen, L., Astrand, P. O., Osted, A., Kongsted, J., and Mikkelsen, K. V. (2002). Polarizability of molecular clusters as calculated by a dipole interaction model. Chem. Phys. 116, 4001-4010. doi: 10.1063/1.1433747

Machetti, F., Bucelli, I., Indiani, G., Kappe, C. O., and Guarna, A. (2007). Parallel synthesis of an amide library based on the 6,8-dioxa-3-azabicyclo[3.2.1] octane scaffold by direct aminolysis of methyl esters. J. Comb. Chem. 9, 454-461. doi: $10.1021 / \mathrm{cc} 060120$ o
O'Connell, K. M. G., Galloway, W. R. J. D., and Spring, D. R. (2013). "the basics of diversity-oriented synthesis," in Diversity-Oriented Synthesis: Basics and Applications in Organic Synthesis, Drug Discovery, and Chemical Biology, ed A. Trabocchi (Hoboken, NJ: John Wiley and Sons), 1-28. doi: 10.1002/9781118618110.ch1

Oksanen, J., Blanchet, F. G., Kindt, R., Legendre, P., Minchin, P. R., O’Hara, R. B., et al. (2016). Vegan: Community Ecology Package. R Package Version 2.3-5.

Spandl, R. J., Bender, A., and Spring, D. R. (2008). Diversity-oriented synthesis; a spectrum of approaches and results. Org. Biomol. Chem. 6, 1149-1158. doi: 10.1039/b719372f

Schenone, M., Dančík, V., Wagner, B. K., and Clemons, P. A. (2013). Target identification and mechanism of action in chemical biology and drug discovery. Nat. Chem. Biol. 9, 232-240. doi: 10.1038/nchembio.1199

Smietana, K., Siatkowski, M., and Møller, M. (2016). Trends in clinical success rates. Nat. Rev. Drug Discov. 15, 379-380. doi: 10.1038/nrd.2016.85

Stefanini, I., Trabocchi, A., Marchi, E., Guarna, A., and Cavalieri, D. (2010). A systems biology approach to dissection of the effects of small bicyclic peptidomimetics on a panel of Saccharomyces cerevisiae mutants. J. Biol. Chem. 285, 23477-23485. doi: 10.1074/jbc.M110.125153

Swinney, D. C. (2013). Phenotypic vs. target-based drug discovery for first-in-class medicines. Clin. Pharmacol. Ther. 93, 299-301. doi: 10.1038/clpt.2012.236

Swinney, D. C., and Anthony, J. (2011). How were new medicines discovered? Nat. Rev. Drug Discov. 10, 507-519. doi: 10.1038/nrd3480

Trabocchi, A., Mancini, F., Menchi, G., and Guarna, A. (2003). A solid-phase approach towards the development of 3-aza-6,8-dioxabicyclo[3.2.1]octane scaffolds. Mol. Divers. 6, 245-250. doi: 10.1023/B:MODI.0000006781.31594.c2

Trabocchi, A., Menchi, G., Guarna, F., Machetti, F., Scarpi, D., and Guarna, A. (2006). Design, synthesis, and applications of 3-Aza-6,8Dioxabicyclo[3.2.1] Octane-based scaffolds for peptidomimetic chemistry. Synlett 3, 331-353. doi: 10.1002/chin.200620261

Trabocchi, A., Occhiato, E. G., Potenza, D., and Guarna, A. (2002). Synthesis and conformational analysis of small peptides containing 6-endo-BT(t)L scaffolds as reverse turn mimetics. J. Org. Chem. 67, 7483-7492. doi: 10.1021/jo0202132

Trabocchi, A., Scarpi, D., and Guarna, A. (2008). Structural diversity of bicyclic amino acids. Amino Acids 34, 1-24. doi: 10.1007/s00726-007-0588-y

Varmuza, K., Filzmoser, P., Liebmann, B., and Dehmer, M. (2012). Redundancy analysis for characterizing the correlation between groups of variables Applied to molecular descriptors. Chemometr. Intell. Lab. Syst. 117, 31-41. doi: 10.1016/j.chemolab.2011.05.013

Venables, W. N., and Ripley, B. D. (2002). Modern Applied Statistics with S, 4th Edn. New York, NY: Springer. doi: 10.1007/978-0-387-21706-2

Wolfger, H., Mamnun, Y. M., and Kuchler, K. (2001). Fungal ABC proteins: pleiotropic drug resistance, stress response and cellular detoxification. Res. Microbiol. 152, 375-389. doi: 10.1016/S0923-2508(01)01209-8

Conflict of Interest Statement: The authors declare that the research was conducted in the absence of any commercial or financial relationships that could be construed as a potential conflict of interest.

Copyright (c) 2017 Brilli, Trabocchi, Weil, Cavalieri and Stefanini. This is an openaccess article distributed under the terms of the Creative Commons Attribution License (CC BY). The use, distribution or reproduction in other forums is permitted, provided the original author(s) or licensor are credited and that the original publication in this journal is cited, in accordance with accepted academic practice. No use, distribution or reproduction is permitted which does not comply with these terms. 